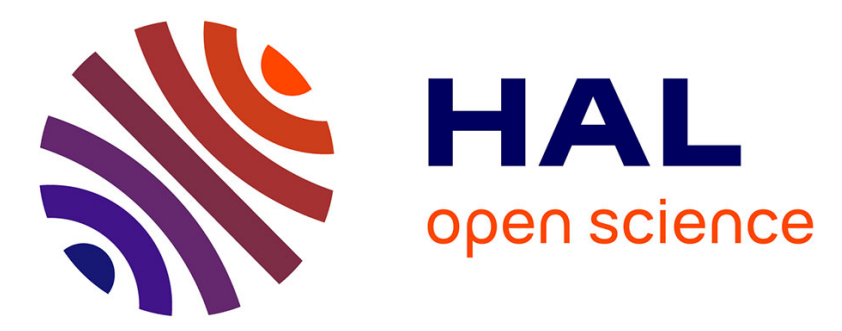

\title{
Control of Delayed Recycling Systems with Unstable First Order Forward Loop
}

Juan-Francisco Márquez-Rubio, Basilio del Muro-Cuellar, M. Velasco-Villa, D. Cortés-Rodríguez, Olivier Sename

\section{- To cite this version:}

Juan-Francisco Márquez-Rubio, Basilio del Muro-Cuellar, M. Velasco-Villa, D. Cortés-Rodríguez, Olivier Sename. Control of Delayed Recycling Systems with Unstable First Order Forward Loop. Journal of Process Control, 2012, 22 (4), pp.729-737. 10.1016/j.jprocont.2012.02.002 . hal-00682111

\section{HAL Id: hal-00682111 \\ https://hal.science/hal-00682111}

Submitted on 23 Mar 2012

HAL is a multi-disciplinary open access archive for the deposit and dissemination of scientific research documents, whether they are published or not. The documents may come from teaching and research institutions in France or abroad, or from public or private research centers.
L'archive ouverte pluridisciplinaire HAL, est destinée au dépôt et à la diffusion de documents scientifiques de niveau recherche, publiés ou non, émanant des établissements d'enseignement et de recherche français ou étrangers, des laboratoires publics ou privés. 


\title{
Control of Delayed Recycling Systems with Unstable First Order Forward Loop
}

\author{
J. F. Márquez-Rubio ${ }^{\mathrm{a}, *}$, B. del Muro-Cuéllar ${ }^{\mathrm{a}}$, M. Velasco-Villa ${ }^{\mathrm{b}}$, D. Cortes ${ }^{\mathrm{a}}$, \\ O. Sename \\ ${ }^{a}$ Instituto Politécnico Nacional, ESIME Unidad Culhuacan, Santa Ana 1000, Coyoacán, \\ D.F., 04430, México \\ ${ }^{b}$ CINVESTAV-IPN, Departamento de Ingeniería Eléctrica, Sección de Mecatrónica, Av. \\ IPN 2508, 07300, México D.F., México. \\ ${ }^{c}$ GIPSA-Laboratory, Automatic Control Department, ENSE3, Domaine \\ Universitaire-BP46, 38402 Saint Martin d'Heres-Cedex, France
}

\begin{abstract}
Unstable time-delay systems and recycling systems are challenging problems for control analysis and design. When an unstable time-delay system has a recycle, its control problem becomes even more difficult. A control methodology for this class of systems is proposed in this paper. The considered strategy is based on the fact that if some internal system signals are available for measurement, then it will be possible to decouple the backward dynamics of the system and then a feedback controller could be designed for the forward dynamics. The key point for this strategy to be carried out is an asymptotic observer-predictor proposed to estimate these required internal signals. Necessary and sufficient conditions to assure convergence of this observer are given. After proving that the proposed control scheme tracks a step input signal and at the same time reject step disturbances, a procedure summarizing the methodology is provided. Robustness with respect to delay uncertainty and model parameters are also analyzed.
\end{abstract}

Keywords: Time-delay, recycling system, stabilization, observer.

\section{Introduction}

In recycling systems, the output of a process is partially fed-back to the input. Recycling processes reuse the energy and the partially processed matter increasing the efficiency of the overall process. They are commonly found in chemical industry, for instance, in order to implement a recycle stream for

\footnotetext{
* Corresponding author

Email addresses: jfcomr23@yahoo.com.mx (J. F. Márquez-Rubio), bdelmuro@yahoo.com (B. del Muro-Cuéllar), velasco@cinvestav.mx (M. Velasco-Villa), domingo.cortes@gmail.com (D. Cortes), olivier.sename@gipsa-lab.grenoble-inp.fr (O. Sename)
} 
a continuous stirred tank reactor (CSTR), the output stream of the reactor is sent through a separation process (perhaps a centrifuge). Then, the unreacted reagents are returned into the CSTR by traveling through pipes. Because recycling reduces waste of reagents, and hence the cost of the reaction, it is widespread used in industry. As another example, recycling is often used in the manufacturing of nylon 66 or in the oxidation of cyclohexene to KA (a mixture of ketone and alcohol of cyclohexene), among many other reactions [1], in a typical plant formed by reactor/separator processes, where reactants are recycled back to the reactor [2], [3].

Recycling processes are systems with positive feedback that can give rise to some undesirable effects. In fact, instability can occur in recycle systems when the feedback gain is larger than unity, as an example, the recycle of the energy developed by an exothermic reaction in an adiabatic plug flow reactor for feed preheating. Instability could occur due to the exponential increase in the reaction rate with the temperature when this cannot be properly controlled [4]. Another example is the recycle of impurities in a plant with recycle, whose inventory cannot be kept at equilibrium by the separator system [5].

Also, the so-called snowball effect is observed in the operation of many chemical plants with recycle streams. Snowball means that a small change in a load variable causes a very large change in the flow rates around the recycle loop. Although snowballing is a steady state phenomenon and has nothing to do with dynamics, it depends on the control structure. Disadvantages of snowball effect has drawn the attention of some researchers proposing several control strategies to avoid the associated problems, [6], [7].

The control of time-delay systems has also been analyzed by the use of observer strategies mainly for systems without time-lag. In the case of systems with delayed-state in [8] it is considered a particular nonlinear triangular system and in [9] a predominant linear system perturbed by a delayed nonlinear term is considered while in [10] an observer adaptive control is proposed. A digital redesign of an analog Smith predictor compensator is developed in [11].

When significant transport delay is present in recycled systems, the control problem becomes more complex. For example, in the case of continuous stirred tank reactors (CSTR), a problem that arises on the recycling loop is that the process is modeled usually by assuming that there is not any time-delay on this path. While this assumption may produce a simpler theoretical analysis, it is highly unrealistic [1].

It is known that in a system with recycle loops and time-delays, exponential terms appears in forward and backward paths on its transfer function representation. In state space representation, recycled systems with time-delay correspond to systems with delays at the input and at the state. Model approximation has been proposed to remove the exponential terms from the transfer function denominator of a delayed system, such as the method of moments [12], and Pade-Taylor approximations [13], [14]. Other techniques, such as the seasonal time-series model [15], have also been proposed to obtain an approximate model. Del Muro et. al. [16] proposed an approximate model to represent recycle systems by using a discrete-time approach. In turn, such approximate 
models can be used for stability analysis or control design [17], [18], [19].

A system with time-delay and open-loop unstable poles is notably more difficult to control than a system with only open-loop stable poles. Introducing recycle in such a system would lead to a very difficult (although interesting)

problem. To begin to overcome this situation, in this work, the problem of a recycled system composed of an unstable first order plant in the direct path and an stable system of order $n$ in the recycle loop is addressed. The control strategy will be based on a particular observer-based structure. A preliminary analysis of this problem was presented at [20].

The rest of the paper is organized as follows: In Section 2 the problem to be tackled is formulated for the class of systems considered in this work and the general idea of its solution is outlined. At this point, the need of an observer-predictor arises. Section 3 presents the proposed control scheme. First, a preliminary result concerning the stability of a class of input-delay systems is presented, then a scheme to estimate some internal signals of the system is proposed. Based on the estimation of these necessary internal variables, the overall control scheme is presented in the last part of Section 3. As robustness of this kind of controllers is a fundamental issue, Section 4 is dedicated to its analysis. Some simulations results are provided in Section 5 in order to illustrate the performance of the proposed control strategy. Finally Section 6 presents some conclusions.

\section{Problem formulation}

Consider the class of recycling system shown in Figure 1, which can be described as,

$$
Y(s)=\left[\begin{array}{ll}
G_{d} & G_{d} G_{r}
\end{array}\right]\left[\begin{array}{c}
U(s) \\
Y(s)
\end{array}\right]
$$

with,

$$
\begin{aligned}
G_{d} & =G_{1}(s) e^{-\tau_{1} s}=\frac{b}{s-a} e^{-\tau_{1} s} \\
G_{r} & =G_{2}(s) e^{-\tau_{2} s}=\frac{N_{r}(s)}{D_{r}(s)} e^{-\tau_{2} s}
\end{aligned}
$$

where $G_{d}(s)$, and $G_{r}(s)$ are transfer functions of the forward (direct) and backward (recycle) paths, respectively; $\tau_{1}, \tau_{2} \geq 0$ are the time-delays associated to $G_{d}(s)$, and $G_{r}(s) . a, b \in \mathbb{R}$, with $a>0$, this is, $G_{d}$ is considered unstable; $N_{r}(s)$ and $D_{r}(s)$ are polynomials on the complex variable $s . U(s)$ is the process input and $Y(s)$ is the process output.

The closed-loop transfer function of system (1) is given by

$$
G_{t}(s)=\frac{D_{r}(s) b e^{-\tau_{1} s}}{(s-a) D_{r}(s)-b N_{r}(s) e^{-\left(\tau_{1}+\tau_{2}\right) s}}
$$




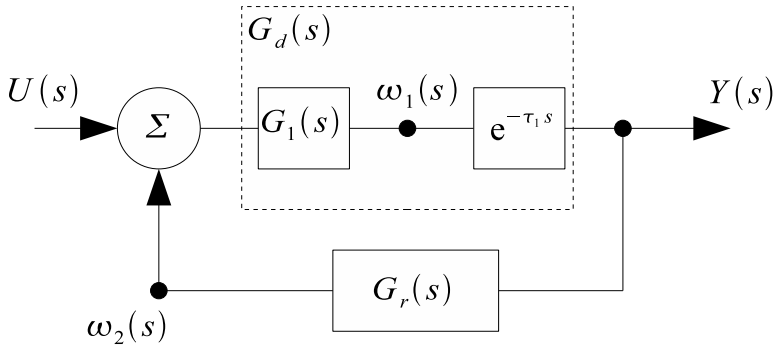

Figure 1: A process with recycle.

Note that exponential terms appear explicitly in numerator and denominator of $G_{t}(s)$. Stability of (3) is determined by the roots of its characteristic equation,

$$
Q(s)=(s-a) D_{r}(s)-b N_{r}(s) e^{-\left(\tau_{1}+\tau_{2}\right) s}=0
$$

More precisely, the overall path $U(s) \rightarrow Y(s)$ is stable if and only if all the roots of $Q(s)$ are contained in the open left-half complex plane. It is well known that the transcendental term in $Q(s)$ induces an infinite number of roots preventing the use of classical control design techniques and standard stability analysis methods.

Let us describe some ideas behind the proposed methodology. Consider an input reference $R(s)$ and a new control law $R_{1}(s)$. Then, with respect to Figure 1 , if signal $\omega_{2}$ is known, then we can set

$$
U(s)=R_{1}(s)-\omega_{2}(s)
$$

obtaining the system shown in Figure 2. At this point, it is possible to design $R_{1}(s)$ as $R_{1}(s)=J(s)\left(R(s)-\omega_{1}(s)\right)$ as shown in Figure 3 and where $J(s)$ is a controller based on the delay free forward model. Since $\omega_{1}$ and $\omega_{2}$ are internal signals of the system, an observer-predictor scheme to estimate these variables is developed in the following section.

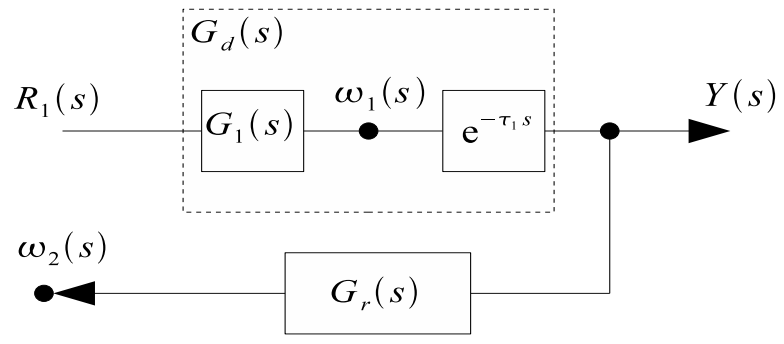

Figure 2: System of Figure 1 after applying U(s) given in (5). 


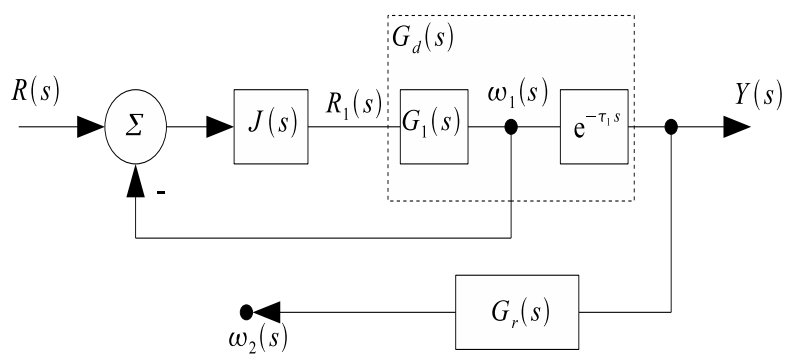

Figure 3: Control structure for the system of Figure 2.

\section{Observer-predictor based control}

\subsection{Preliminary results}

In this section, a well known stability condition for an unstable first order plus time-delay system is recalled from [21] for the sake of completeness. This result will be used later in the proof of the observer-predictor convergence.

Lemma 1. Consider the unstable input-output delay system

$$
\frac{Y(s)}{U(s)}=G(s) e^{-\tau s}=\frac{b}{s-a} e^{-\tau s}, \quad a>0
$$

with a proportional output feedback

$$
U(s)=R(s)-k Y(s)
$$

where $R(s)$ is the new reference input. There exist a proportional gain $k$ such that the closed-loop system

$$
\frac{Y(s)}{R(s)}=\frac{b e^{-\tau s}}{s-a+k b e^{-\tau s}}
$$

is stable if and only if $\tau<1 / a$.

Stability of (8) has been previously studied in the literature. Lemma 1 can be proved using a classical frequency domain; D-decomposition or even by the classical Pontryagin Method [22], [23], [24]. An alternative proof of Lemma 1 is provided in [21] by using a discrete-time approach and is included in Appendix A.

A useful practical result in order to compute the parameter $k$ involved on the control scheme is as follows.

Corollary 1. [21] Consider system given by (6) with $\tau<1 / a$. Then, there exists $k \in R^{+}$that stabilizes the closed-loop system (8), satisfying $\alpha<k<\beta$, with $\alpha=a / b$ and some constant $\beta>a / b$.

The proof of Corollary 1 is also presented in Appendix A. 
Remark 1. From a frequency domain analysis, it is not difficult to accurately determinate the value of $\beta$ given in Corollary 1. In fact, such value is given by $\beta=\frac{a}{b} \sqrt{1+\left(\frac{\omega}{a}\right)^{2}}$, where $\omega$ satisfy $\frac{\omega}{a}=\tan (\omega \tau)$ for $0<\omega<\frac{\pi}{2 \tau}$. The utility of Corollary 1 comes from the fact that any $k=\frac{a}{b}+\epsilon$, with $\epsilon>0$ stabilizes the closed-loop system (8) for $\epsilon$ sufficiently small. Notice that the upper bound $\beta$ would depend directly on the values of the parameters $a, b$ and $\tau$ and therefore it is determined by the considered plant parameters

\subsection{Prediction Strategy}

In order to estimate signals $\omega_{1}$ and $\omega_{2}$ shown in Figure 1, the observerpredictor scheme depicted in Figure 4 is proposed. Its convergence is established in the following result.

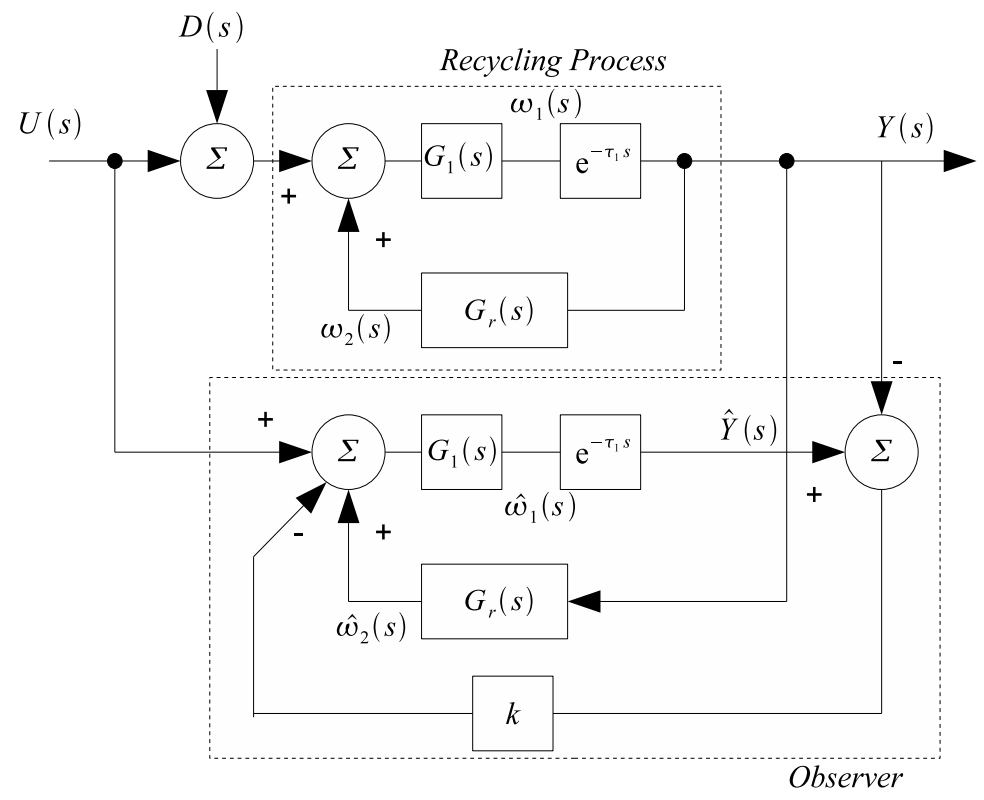

Figure 4: Proposed observer schema.

Theorem 1. Consider the observer-predictor scheme shown in Figure 4, with $G_{r}$ a delayed stable transfer function. Then, there exists a constant $k$ such that

$$
\lim _{t \rightarrow \infty}\left[\omega_{i}-\widehat{\omega}_{i}\right]=0, \text { for } i=1,2,
$$

if and only if $\tau_{1}<\frac{1}{a}$.

Proof. A state space representation of the observer-predictor scheme shown in Figure 4 is

$$
\begin{aligned}
& \dot{\mathbf{x}}(t)=A \mathbf{x}(t)+A_{1} \mathbf{x}\left(t-\tau_{1}\right)+A_{2} \mathbf{x}\left(t-\tau_{2}\right)+B u(t) \\
& \mathbf{y}(t)=C_{1} \mathbf{x}\left(t-\tau_{1}\right)
\end{aligned}
$$


with,

$$
\begin{aligned}
& \mathbf{x}(t)=\left[\begin{array}{llll}
x_{d}(t) & x_{r}(t) & \widehat{x}_{d}(t) & \widehat{x}_{r}(t)
\end{array}\right]^{T} \\
& \mathbf{y}(t)=\left[\begin{array}{ll}
y(t) & \widehat{y}(t)
\end{array}\right]^{T}, B=\left[\begin{array}{llll}
B_{d} & 0 & B_{d} & 0
\end{array}\right]^{T} \\
& A=\left[\begin{array}{cccc}
A_{d} & 0 & 0 & 0 \\
0 & A_{r} & 0 & 0 \\
0 & 0 & A_{d} & 0 \\
0 & 0 & 0 & A_{r}
\end{array}\right], \quad A_{1}=\left[\begin{array}{cccc}
0 & 0 & 0 & 0 \\
B_{r} C_{d} & 0 & 0 & 0 \\
B_{r} k C_{d} & 0 & -B_{d} k C_{d} & 0 \\
B_{r} C_{d} & 0 & 0 & 0
\end{array}\right] \\
& A_{2}=\left[\begin{array}{cccc}
0 & B_{d} C_{r} & 0 & 0 \\
0 & 0 & 0 & 0 \\
0 & 0 & 0 & B_{d} C_{r} \\
0 & 0 & 0 & 0
\end{array}\right], \quad C_{1}=\left[\begin{array}{cccc}
C_{d} & 0 & 0 & 0 \\
0 & 0 & C_{d} & 0
\end{array}\right]
\end{aligned}
$$

where $\mathbf{x} \in \mathbb{R}^{n}$ is the state vector, $u \in \mathbb{R}$ is the input, $\mathbf{y} \in \mathbb{R}^{2}$ is the output, $\tau_{1} \geq 0$ and $\tau_{2} \geq 0$ are the time-delays present in the system. $A_{d} \in \mathbb{R}^{n \times n}, B_{d} \in \mathbb{R}^{n \times 1}$, and $C_{d} \in \mathbb{R}^{1 \times n}$ are matrices and vectors parameters that corresponds to the forward loop in the process, and $A_{r} \in \mathbb{R}^{m \times m}, B_{r} \in \mathbb{R}^{m \times 1}$, and $C_{r} \in \mathbb{R}^{1 \times m}$ are matrices and vectors parameters that corresponds to backward path in the process, $\widehat{x}(t)$ is the estimation of $x(t)$.

Defining the state prediction errors

$$
e_{x_{d}}(t)=\widehat{x}_{d}(t)-x_{d}(t), \quad e_{x_{r}}(t)=\widehat{x}_{r}(t)-x_{r}(t),
$$

and the output estimation

$$
e_{y}(t)=\widehat{y}(t)-y(t)
$$

it is possible to describe the behavior of the error signals as,

$$
\left[\begin{array}{c}
\dot{e}_{x_{d}}(t) \\
\dot{e}_{x_{r}}(t) \\
e_{y}\left(t+\tau_{1}\right) \\
e_{\omega_{2}}\left(t+\tau_{2}\right)
\end{array}\right]=\left[\begin{array}{cccc}
A_{d} & 0 & -B_{d} k & B_{d} \\
0 & A_{r} & 0 & 0 \\
C_{d} & 0 & 0 & 0 \\
0 & C_{r} & 0 & 0
\end{array}\right]\left[\begin{array}{c}
e_{x_{d}}(t) \\
e_{x_{r}}(t) \\
e_{y}(t) \\
e_{\omega_{2}}(t)
\end{array}\right]
$$

Note that $e_{y}(t)=C_{d} e_{x_{d}}\left(t-\tau_{1}\right)$ and that $e_{\omega_{2}}(t)=C_{r} e_{x_{r}}\left(t-\tau_{2}\right)$. Then, system (14) can be rewritten as

$$
\begin{aligned}
& \dot{e}_{x_{d}}(t)=A_{d} e_{x_{d}}(t)-B_{d} k C_{d} e_{x_{d}}\left(t-\tau_{1}\right)+B_{d} C_{r} e_{x_{r}}\left(t-\tau_{2}\right) \\
& \dot{e}_{x_{r}}(t)=A_{r} e_{x_{r}}(t)
\end{aligned}
$$

Since $A_{r}$ is a Hurwitz matrix, the stability of system (15) can be analyzed by considering the partial dynamics

$$
\dot{e}_{x_{d}}(t)=A_{d} e_{x_{d}}(t)-B_{d} k C_{d} e_{x_{d}}\left(t-\tau_{1}\right)
$$

or equivalently,

$$
\left[\begin{array}{c}
\dot{e}_{x_{d}}(t) \\
e_{y}\left(t+\tau_{1}\right)
\end{array}\right]=\left[\begin{array}{cc}
A_{d} & -B_{d} k \\
C_{d} & 0
\end{array}\right]\left[\begin{array}{c}
e_{x_{d}}(t) \\
e_{y}(t)
\end{array}\right]
$$


Consider now a state space realization of system (8). It is easy to see that this dynamics can be written in state space form as,

$$
\left[\begin{array}{c}
\dot{x}_{d}(t) \\
y\left(t+\tau_{1}\right)
\end{array}\right]=\left[\begin{array}{cc}
A_{d} & -B_{d} k \\
C_{d} & 0
\end{array}\right]\left[\begin{array}{c}
x_{d}(t) \\
y(t)
\end{array}\right]+\left[\begin{array}{c}
B_{d} \\
0
\end{array}\right] u(t)
$$

Comparing (18) and (17) it is clear that Lemma 1 can be applied to system (17). Hence, the result of the theorem follows.

Remark 2. A natural step toward a more general result is the extension of Lemma 1 to a higher order unstable system. In [25] it is presented the stabilization of delayed systems with one unstable pole and $n$ stable poles. In the case that $n=1$, the stability condition become $\tau_{1}<\frac{1}{a}-\frac{1}{b}$, with $b$ as the position of the stable pole. Notice that under these circumstances, it is possible to construct an observer-predictor for this more general system that will produce an error dynamics equivalent to the one described in equation (16). Notice that in the general case, this is, the consideration of an unstable transfer function on the forward path and a stable one on the recycle path together with a time-delay on the forward path it is also possible to construct an observer-predictor that will produce an error dynamics as in (16). The remaining problem consist in finding stabilization conditions in terms of the obtained $A_{d}$ and $B_{d} k C_{d}$ that in the present case, can be accomplished due to the lower dimension of the problem.

\subsection{Proposed control scheme}

Once the estimated internal signals $\widehat{\omega}_{1}$ and $\widehat{\omega}_{2}$ converge to their actual values, the ideas described in Section 2 can be implemented. The complete control strategy, proposed in this work, is depicted in Figure 5. In what follows, it will be shown that the proposed scheme achieves step input tracking and a particular disturbance rejection action by using a PI controller with two degree of freedom. With this aim, consider first the general control strategy depicted in Figure 5, given by,

$$
U(s)=J(s)\left(R(s)-\hat{\omega}_{1}+E_{y}(s)\right)-\hat{\omega}_{2}(s)
$$

Then, in order to improve the output response performance, consider a PI controller with two degree of freedom provided in [26], instead of a simple controller $J(s)$. In such case, the control law can be implemented as,

$$
U(s)=R(s) G_{f f}(s)-G_{c}(s)\left(\widehat{\omega}_{1}(s)-E_{y}(s)\right)-\widehat{\omega}_{2}(s)
$$

where,

$$
\begin{aligned}
G_{f f}(s) & =K_{p}\left(\sigma+\frac{1}{T_{i} s}\right) \\
G_{c}(s) & =K_{p}\left(1+\frac{1}{T_{i} s}\right)
\end{aligned}
$$

The following results are concerned with the step tracking reference and the step disturbance rejection problem by considering a PI controller with two degree of freedom for the proposed observer strategy. 


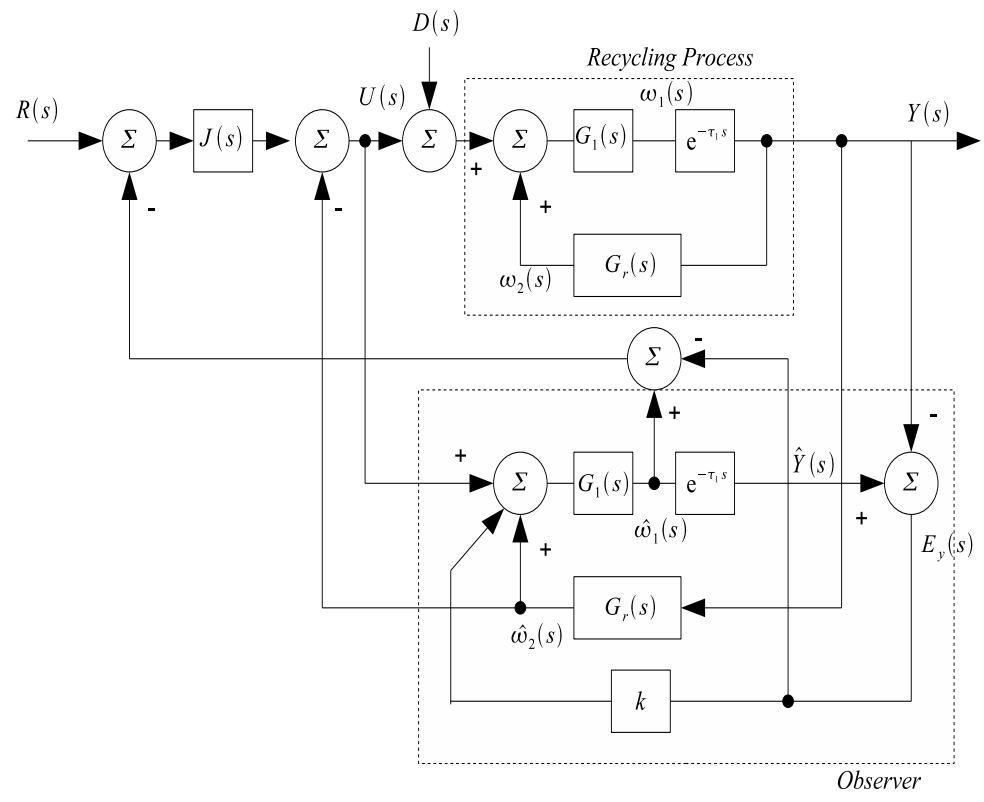

Figure 5: Proposed control schema.

Lemma 2. Consider the observer scheme shown in Figure 5. Then, there exists a PI controller with two degree of freedom given by (20) such that $\lim _{t \rightarrow \infty} y(t)=$ $\alpha$, where $R(s)=\alpha / s$ is the step input reference and $D(s)=0$.

Proof. Consider the observer scheme shown in Figure 5 and the control strategy given by (20). Then,

$$
\frac{Y(s)}{R(s)}=\frac{G_{d} G_{f f}\left(1+k G_{1} e^{-s \tau_{1}}\right)}{G_{1} G_{c}+G_{c} G_{d}-G_{1} G_{c} e^{-s \tau_{1}}+k G_{1} e^{-s \tau_{1}}+k G_{1} G_{c} G_{d}+1}
$$

Applying the Final Value Theorem with $R(s)=\alpha / s$, as input reference to the transfer function (22), it is produced,

$$
\lim _{t \rightarrow \infty} y(t)=\lim _{s \rightarrow 0} s Y(s)=\lim _{s \rightarrow 0} s \frac{N_{1}(s)}{D_{1}(s)} \frac{\alpha}{s}
$$

with

$$
\begin{gathered}
N_{1}(s)=G_{d} G_{f f}\left(1+k G_{1} e^{-s \tau_{1}}\right) \\
D_{1}(s)=G_{1} G_{c}+G_{c} G_{d}-G_{1} G_{c} e^{-s \tau_{1}}+k G_{1} e^{-s \tau_{1}}+k G_{1} G_{c} G_{d}+1
\end{gathered}
$$

Substituting $G_{d}, G_{1}$ given in (2a) and the controller $G_{f f}$ and $G_{c}$ given in (21), we have,

$$
\lim _{t \rightarrow \infty} y(t)=\lim _{s \rightarrow 0} s Y(s)=\alpha
$$


Lemma 3. Consider the proposed observer scheme shown in Figure 5. Then, there exist a PI controller with two degree of freedom provided by (20) such that $\lim _{t \rightarrow \infty} y(t)=0$, where $R(s)=0$ and $D(s)$ is a step input disturbance.

Proof. According to Figure 5,

$$
\frac{Y(s)}{D(s)}=\frac{G_{d}+G_{1} G_{c} G_{d}+k G_{1} G_{d} e^{-s \tau_{1}}-G_{1} G_{c} G_{d} e^{-s \tau_{1}}}{G_{1} G_{c}+G_{c} G_{d}-G_{1} G_{c} e^{-s \tau_{1}}+k G_{1} e^{-s \tau_{1}}+k G_{1} G_{c} G_{d}+1}
$$

Applying the Final Value Theorem with $D(s)=\eta / s$, as input reference. From equation (22), we have,

$$
\lim _{t \rightarrow \infty} y(t)=\lim _{s \rightarrow 0} s Y(s)=\lim _{s \rightarrow 0} s \frac{N_{2}(s)}{D_{2}(s)} \frac{\eta}{s}
$$

with

$$
\begin{gathered}
N_{2}(s)=G_{d}+G_{1} G_{c} G_{d}+k G_{1} G_{d} e^{-s \tau_{1}}-G_{1} G_{c} G_{d} e^{-s \tau_{1}} \\
D_{2}(s)=G_{1} G_{c}+G_{c} G_{d}-G_{1} G_{c} e^{-s \tau_{1}}+k G_{1} e^{-s \tau_{1}}+k G_{1} G_{c} G_{d}+1 .
\end{gathered}
$$

Substituting $G_{d}, G_{1}$ given in (2a) and the controller $G_{f f}$ and $G_{c}$ given in (21), we have,

$$
\lim _{t \rightarrow \infty} y(t)=\lim _{s \rightarrow 0} s Y(s)=0
$$

From the previous discussions and results, the proposed methodology can be summarized as follows:

1. Make sure that the conditions of Theorem 1 are satisfied, that is, $G_{r}(s)$ a stable transfer function and $\tau_{1}<\frac{1}{a}$ for the unstable first order delayed plant.

2. Tune the forward loop with the parameter $k$ using Corollary 1.

3. Design of a controller $J(s)$ based on the delay free model of the forward path. A PI or PID control based strategy can be considered.

4. Finally, implement the general control structure as it is shown in Figure 5.

\section{Robustness with respect to uncertainties}

The control strategy presented in previous sections has been developed under the assumption that an accurate model of the process is available. In observerbased controllers for time-delay and recycled systems, it is important to analyze the robustness of the closed-loop system, under plant parameters and timedelays uncertainties. This analysis is carried out in this Section for the proposed controller. 
Consider a state representation of the open-loop system with recycle in the nominal case (which can be obtained from the complete state representation of system-observer expressed in equation (10)),

$$
\begin{aligned}
\dot{x} & =\bar{A} x+\bar{A}_{1} x\left(t-\tau_{1}\right)+\bar{A}_{2} x\left(t-\tau_{2}\right)+\bar{B} u(t) \\
y & =\bar{C}_{1} x\left(t-\tau_{1}\right)
\end{aligned}
$$

where

$$
\begin{gathered}
x=\left[\begin{array}{ll}
x_{d} & x_{r}
\end{array}\right]^{T}, \quad \bar{A}=\left[\begin{array}{cc}
A_{d} & 0 \\
0 & A_{r}
\end{array}\right], \quad \overline{A_{1}}=\left[\begin{array}{cc}
0 & 0 \\
B_{r} C_{d} & 0
\end{array}\right], \\
\overline{A_{2}}=\left[\begin{array}{cc}
0 & B_{d} C_{r} \\
0 & 0
\end{array}\right], \quad \bar{B}=\left[\begin{array}{c}
B_{d} \\
0
\end{array}\right], \quad \bar{C}_{1}=\left[\begin{array}{ll}
C_{d} & 0
\end{array}\right]
\end{gathered}
$$

Before define perturbations for the recycling system, as an example, let the nominal system be,

$$
\dot{x}(t)=A x(t)+B x(t-\tau),
$$

and the actual system as above but with $\tau$ replaced by $\tau_{0}$, then $p(x(t))=$ $B\left[x\left(t-\tau_{0}\right)-x(t-\tau)\right]$. In this way, with $\theta=\tau_{0}-\tau$, we get,

$$
P_{1}(s, \theta)=B\left[e^{-\tau_{0} s}-e^{-\tau s}\right]=B e^{-\tau s}\left[e^{-\theta s}-1\right] .
$$

Under the same idea, it is also possible to consider uncertainties on the matrix $B$. Let us consider the actual input matrix system as $B_{0}$, then $p(x(t))=B[x(t-$ $\left.\left.\tau_{0}\right)-x(t-\tau)\right]+\left[B_{0}-B\right] x\left(t-\tau_{0}\right)$. Assuming $B_{\delta}=B_{0}-B$, it is obtained,

$$
P_{2}(s, \theta, \delta)=e^{-\tau s}\left[B\left(e^{-\theta s}-1\right)+B_{\delta} e^{-\theta s}\right] .
$$

Now, let define perturbations on the time-delays for the recycling system (30). The nominal values of the time-delays are $\tau_{1}$ and $\tau_{2}$. Thus, the delay uncertainty is obtained as follows,

$$
\begin{aligned}
& P(s, \theta)=\bar{A}_{1} e^{-s \tau_{1}}\left(e^{-s \theta_{1}}-1\right)+\bar{A}_{2} e^{-s \tau_{2}}\left(e^{-s \theta_{2}}-1\right), \\
& Q(s, \theta)=\bar{C}_{1} e^{-s \tau_{1}}\left(e^{-s \theta_{1}}-1\right) .
\end{aligned}
$$

Notice that the uncertainties expressed in equations (32a)-(32b) allows to analyze the plant uncertainty in both, direct and recycle paths. Furthermore, the uncertainties can be acting with different proportion due to its independent characterization as $\theta_{1}$ and $\theta_{2}$. Also, independent robustness analysis for each time-delay $\tau_{1}$ or $\tau_{2}$ is possible (this can be done by removing $\tau_{2}$ or $\tau_{1}$ from equations (32a) or (32b), respectively).

In the case that uncertainties are present on both time-delays and matrices $\bar{A}, \bar{A}_{1}$ and $\bar{A}_{2}$, it is obtained,

$$
\begin{aligned}
P(s, \theta, \delta)= & \bar{A}_{\delta}+e^{-s \tau_{1}}\left[\bar{A}_{1}\left(e^{-s \theta_{1}}-1\right)+\bar{A}_{1 \delta_{1}} e^{-s \theta_{1}}\right]+ \\
& e^{-s \tau_{2}}\left[\bar{A}_{2}\left(e^{-s \theta_{2}}-1\right)+\bar{A}_{2 \delta_{2}} e^{-s \theta_{2}}\right], \\
Q(s, \theta)= & \bar{C}_{1} e^{-s \tau_{1}}\left(e^{-s \theta_{1}}-1\right) .
\end{aligned}
$$


where, $\bar{A}, \overline{A_{1}}$ and $\overline{A_{2}}$ are the nominal matrices of the recycle system and the corresponding uncertainties are given as $\bar{A}_{\delta}, \bar{A}_{1 \delta_{1}}$ and $\bar{A}_{2 \delta_{2}}$.

A state space representation in the Laplace domain of the observer-based control structure shown in Figure 5 can be expressed as,

$$
\mathrm{sX}(\mathrm{s})=\mathcal{A X}(\mathrm{s})+\mathcal{A}_{1} e^{-s \tau_{1}} \mathrm{X}(\mathrm{s})+\mathcal{A}_{2} e^{-s \tau_{2}} \mathrm{X}(\mathrm{s})+\mathcal{B} R(s)
$$

with $\mathrm{X}(\mathrm{s})=\left[\begin{array}{ll}e_{x}(s) & X(s)\end{array}\right]^{T}, e_{x}(s)=\widehat{X}(s)-X(s)$,

$$
\begin{gathered}
\mathcal{A}=\left[\begin{array}{cc}
\bar{A} & G Q-P \\
-\bar{B} J \bar{K} & \bar{A}-\bar{B} J \bar{K}+P-\bar{B} J Q
\end{array}\right], \\
\mathcal{A}_{1}=\left[\begin{array}{cc}
\bar{A}_{1}-G \bar{C}_{1} & 0 \\
\bar{B} J \bar{C}_{1} & \bar{A}_{1}
\end{array}\right], \mathcal{A}_{2}=\left[\begin{array}{cc}
\bar{A}_{2} & 0 \\
-\bar{B} L & \bar{A}_{2}-\bar{B} L
\end{array}\right], \mathcal{B}=\left[\begin{array}{c}
0 \\
\bar{B} J
\end{array}\right] .
\end{gathered}
$$

For simplicity of notation, in what follows, the simplest case where $G_{d}$ and $G_{r}$ are first order plants will be analyzed. This fact produces,

$$
G=\left[\begin{array}{ll}
B_{d} k & B_{r}
\end{array}\right]^{T}, \quad \bar{K}=\left[\begin{array}{ll}
1 & 0
\end{array}\right], \quad L=\left[\begin{array}{ll}
0 & C_{r}
\end{array}\right] .
$$

The characteristic equation of system (34), is given by,

$$
\gamma=\operatorname{det}\left(s I-\mathcal{A}-\mathcal{A}_{1} e^{-\tau_{1} s}-\mathcal{A}_{2} e^{-\tau_{2} s}\right)
$$

this is,

$$
\begin{aligned}
\gamma & =\operatorname{det}\left[\begin{array}{cc}
s I-F+G \bar{C}_{1} e^{-\tau_{1} s} & P-G Q \\
M-\bar{B} J \bar{C}_{1} e^{-\tau_{1} s} & s I-F+M+\bar{B} J Q-P
\end{array}\right] \\
& =\operatorname{det}\left(s I-F+G \bar{C}_{1} e^{-\tau_{1} s}\right) \operatorname{det}(s I-F+M) \operatorname{det}\left(I+\psi^{-1} \Theta(s, \theta)\right)=0
\end{aligned}
$$

where

$$
\begin{aligned}
F & =\bar{A}+\bar{A}_{1} e^{-\tau_{1} s}+\bar{A}_{2} e^{-\tau_{2} s}, \\
M & =\bar{B} J \bar{K}+\bar{B} L e^{-\tau_{2} s},
\end{aligned}
$$

and

$$
\psi=\left[\begin{array}{cc}
s I-F+G \bar{C}_{1} e^{-\tau_{1} s} & 0 \\
M-\bar{B} J \bar{C}_{1} e^{-\tau_{1} s} & s I-F+M
\end{array}\right]
$$

is the matrix corresponding to the combined observer-controller for nominal system, and $\Theta(s ; \theta)$ collects the plant uncertainties.

Considering that the closed-loop quasi polynomials $\operatorname{det}\left(s I-F+G \bar{C}_{1} e^{-\tau_{1} s}\right)$ and $\operatorname{det}(s I-F+M)$ are stable for a proper choice of $G, \bar{K}$ and $L$, then they do not change sign when $s$ sweeps the imaginary axis and the perturbed closed-loop system remains stable if $\operatorname{det}\left(I+\psi^{-1} \Theta(s ; \theta)\right)$ does not change sign for all $s=j \omega$.

Straightforward computations produce,

$$
\begin{aligned}
\operatorname{det}\left(I+\psi^{-1} \Theta(s, \theta)\right) & =\operatorname{det}\left[I+\left[\begin{array}{ll}
\widetilde{Q}_{p q} & \widetilde{Q}_{p}
\end{array}\right]\left[\begin{array}{c}
P-G Q \\
\bar{B} J Q-P
\end{array}\right]\right] \\
& =\operatorname{det}\left[I+N_{c}(s) D_{c}(s)\right]
\end{aligned}
$$


where,

$$
\begin{aligned}
\widetilde{Q}_{p q} & =-(s I-F+M)^{-1}\left(M-\bar{B} J \bar{C}_{1} e^{-\tau_{1} s}\right)\left(s I-F+G \bar{C}_{1} e^{-\tau_{1} s}\right)^{-1}, \\
\widetilde{Q}_{p} & =(s I-F+M)^{-1},
\end{aligned}
$$

which only depends on the nominal system and observer/controller parameter. By using Rouche's theorem [27], it follows that the closed-loop stability condition for the perturbed system results,

$$
\left\|N_{c}(s) D_{c}(s, \theta)\right\|_{\infty}<1 .
$$

Therefore, the considered controller-observer strategy for the actual perturbed system (34) preserves the closed-loop stability for all uncertainties $\theta_{1}, \theta_{2}, \bar{A}_{\delta}$, $\bar{A}_{1 \delta_{1}}$ and $\bar{A}_{2 \delta_{2}}$ satisfying condition (44).

Remark 3. The consideration of the Rouche's Theorem in the analysis of uncertain linear delayed systems has been previously reported in [28], [29], [30] in the case of time-delay uncertainties. A similar condition has also been proposed in the literature in terms of the singular values of the associated matrices [31], [32] in the analysis of structured uncertainties.

\section{Simulation results}

In this section, the performance of the observer-based control strategy proposed previously is evaluated by means of two numerical examples.

\section{Example 1}

Let us consider a simple reaction $A \rightarrow B$ in a reactor-separator system with recycle of the type discussed in [33] and including time-delays at direct and forward loops. In this way, the open-loop linear model can be written in the form of (30) where the state $x_{d}$ is the reactor temperature $(T)$ and $x_{r}$ is the component concentration $\left(C_{A}\right)$. The manipulated input is the jacket reactor temperature $\left(T_{j}\right)$. Also, the involved constant matrices are given by,

$$
\begin{aligned}
\bar{A} & =\left[\begin{array}{cc}
\frac{F}{V}(1-\lambda)-\frac{F}{V}-\frac{U A}{V \rho c_{p}}+\frac{(-\Delta H)}{\rho c_{p}} C_{A s} k_{p s} & 0 \\
0 & \bar{F}(1-\lambda)-\frac{F}{V}+k_{s}
\end{array}\right], \\
\bar{A}_{1} & =\left[\begin{array}{cc}
0 & 0 \\
C_{A s} k_{p s} & 0
\end{array}\right], \bar{A}_{2}=\left[\begin{array}{cc}
0 & 0 \\
C_{A s} k_{p s} & 0
\end{array}\right], \bar{B}=\left[\begin{array}{c}
\frac{U A}{V \rho c_{p}} \\
0
\end{array}\right]
\end{aligned}
$$

where $k_{s}=k_{0} \exp \left(-E_{a} / R T_{s}\right)$ and $k_{p s}=k_{s}\left(E_{a} / R T_{s}^{2}\right)$. It is assumed that $\tau_{1}$ is the time-delay due to temperature measurement and $\tau_{2}$ the time lag caused by physical transport. The output matrix is $\bar{C}_{1}=\left[\begin{array}{ll}1 & 0\end{array}\right]$, since temperature (first state) is the measured signal. The transfer functions of direct and recycle paths are given by,

$$
G_{d}=\frac{\bar{B}(1,1)}{s+\bar{A}(1,1)} e^{-\tau_{1} s}, \quad G_{r}=\frac{\bar{B}(1,1)^{-1} \bar{A}_{1}(2,1) \bar{A}_{2}(1,2)}{s+\bar{A}(2,2)} e^{-\tau_{2} s}
$$




\begin{tabular}{ll}
\hline \hline Operating Volume $(V)$ & $500 \mathrm{ft}^{3}$ \\
Operating Flowrate $(F)$ & $2000 \mathrm{ft} \mathrm{t}^{3} / \mathrm{hr}$ \\
Reactor Diameter $\left(D_{r}\right)$ & $7.5 \mathrm{ft}$ \\
Overall heat-transfer coefficient $(U)$ & $492.3192 \mathrm{Btu} /\left(\mathrm{hr} f \mathrm{ft}^{2}{ }^{\circ} \mathrm{F}\right)$ \\
Heat transfer area through reactor wall $(A)$ & $47.1238 \mathrm{ft}^{2}$ \\
Preexponential factor $\left(k_{0}\right)$ & $16.96 x 10^{12} \mathrm{hr}^{-1}$ \\
Activation energy $\left(E_{a}\right)$ & $32400 \mathrm{Btu} / \mathrm{lbmol}$ \\
Ideal gas constant $(R)$ & $1.987 \mathrm{Btu} / \mathrm{lbmol}{ }^{\circ} \mathrm{F}$ \\
Heat of reaction $(-\Delta H)$ & $39000 \mathrm{Btu} / \mathrm{lbmol} \mathrm{PO}$ \\
Density of coolant $(\rho)$ & $53.25 \mathrm{lb} / \mathrm{ft} \mathrm{t}^{3}$ \\
Heat capacity of coolant $\left(c_{p}\right)$ & $1 \mathrm{Btu} /\left(\mathrm{lb} \mathrm{b}^{\circ} \mathrm{F}\right)$ \\
Operating concentration $\left(C_{A s}\right)$ & $0.066 \mathrm{lbmol} / \mathrm{ft}$ \\
Operating temperature $\left(T_{s}\right)$ & $560.77^{\circ} R$ \\
Forward loop time-delay $\left(\tau_{1}\right)$ & $0.1 \mathrm{hr}$ \\
Backward loop time-delay $\left(\tau_{2}\right)$ & $0.2 \mathrm{hr}$ \\
Recirculation coefficient $(\lambda)$ & 0.5 \\
\hline \hline
\end{tabular}

Table 1: Constant parameters for Example 5

The proposed observer-based control strategy given in Figure 5, is implemented by considering the parameters values given in Table 1 . Since conditions of Theorem 1 are satisfied, a set of proportional gains can be calculated as $8.18<$ $k<13.19$ (see Corollary 1 and Remark 1), choosing in consequence $k=9$ for all the experiments. The two degree of freedom PI controller is implemented by considering $K_{p}=41, \sigma=0.5$ and $T_{i}=0.142$.

In order to analyze the robustness of the control strategy with respect to time delays, Figure 6 shows the stability condition given by equation (44), where the uncertainties in time-delays $\theta_{1}=0.012 h r$ and $\theta_{2}=0.04 h r$ are considered. In this case, such combination of uncertainties gives as result a stable closed-loop system since $\left\|N_{c}(s) D_{c}(s, \theta)\right\|_{\infty}=0.9872<1$. Taking into account the timedelay uncertainty mentioned above, in Figure 6 it is also presented the stability condition (44) when all parameters of the model are different from the nominal. In this case, the following uncertainties are considered,

$$
\bar{A}_{\delta}=\left[\begin{array}{cc}
0.03 & 0 \\
0 & 0.08
\end{array}\right], \quad \bar{A}_{1 \delta_{1}}=\left[\begin{array}{cc}
0 & 0 \\
-0.11 & 0
\end{array}\right], \quad \bar{A}_{2 \delta_{2}}=\left[\begin{array}{cc}
0 & 0.025 \\
0 & 0
\end{array}\right] .
$$

As it is seen from Figure 6, this set of uncertainties satisfies the stability condition (44) and therefore the stability of the closed-loop system is also assured.

Now, in order to evaluate the output signal evolution, it is considered a positive unit step input and initial conditions in the process and the observer of magnitude 0.1 and 0.2 units, respectively. In Figure 7, a continuous line shows the output response when it is considered the exact knowledge of the model parameters; a dashed line depicts the output signal when time-delays $\tau_{1}, \tau_{2}$, are increased by $10 \%$ and the nominal direct path unstable pole $s_{1}=7.1318$ is shifted to $s_{1}=7.3$ and the nominal stable recycle-path pole $s_{1}=-6$ is 
considered as $s_{2}=-6.2$. Additionally, a step disturbance $d(t)$ with a magnitude of 0.3 units acting at $3 h r$ is considered. The corresponding control signals are also depicted in the lower part of the figure.

To conclude with this example, Figure 8 shows the corresponding output error $e_{y}(t)$, when it is considered exact knowledge of the model parameters; a positive unit step input and initial conditions in the process and the observer of magnitude 0.1 and 0.2 units, respectively.

It can be seen from Figures 7 and 8 how the observer-based control strategy produces an adequate response of the system.

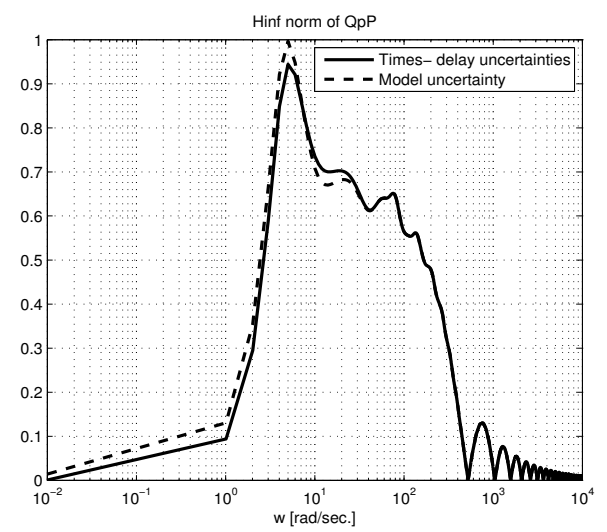

Figure 6: $\left\|N_{c}(s) D_{c}(s, \theta)\right\|_{\infty}$ for $\theta_{1}=0.012$ and $\theta_{2}=0.04$.
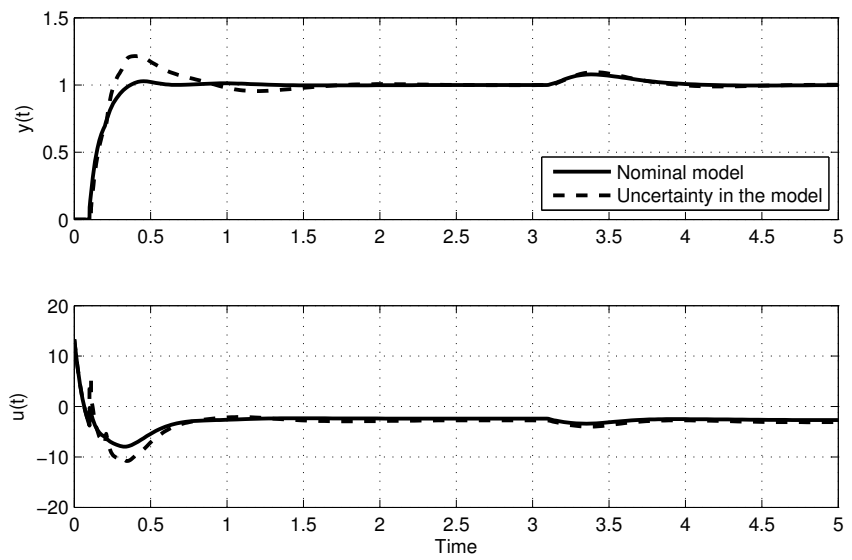

Figure 7: Output and control signals for different initial condition in process and observer, Example 5. 


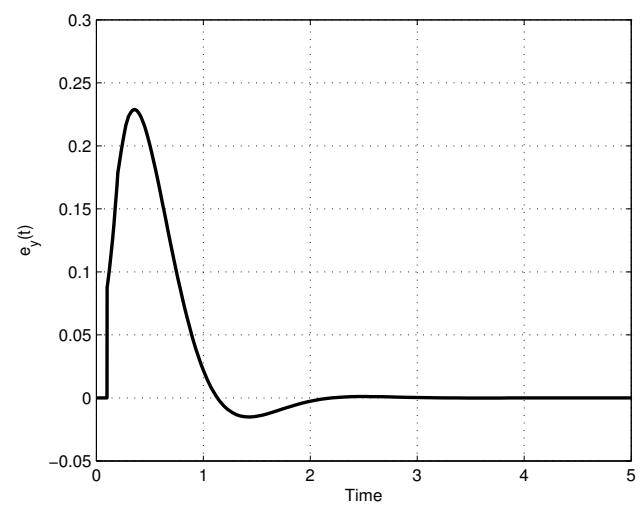

Figure 8: Estimation error $e_{y}(t)$ by considering different initial condition in process and observer, Example 5.

\section{Example 2}

Consider now a recycled system of the form (1) with,

$$
G_{d}=\frac{1}{s-0.25} e^{-2 s}, \quad G_{r}=\frac{10}{(s+1)(s+2)} e^{-2 s} .
$$

In this case, the proportional observer design gain is $k=0.3$, chosen from $0.25<k<0.6342$, the control feedback (20) is obtained by considering,

$$
G_{f f}(s)=1.5\left(0.4+\frac{1}{4 s}\right) \text { and } G_{c}(s)=1.5\left(1+\frac{1}{4 s}\right)
$$

Consider now time-delays uncertainties of $\theta_{1}=0.016$ and $\theta_{2}=0.05$. Thus, the stability condition given by (44) is shown in Figure 9. As it is seen, such combination of uncertainties produces a stable closed-loop operation since

$$
\left\|N_{c}(s) D_{c}(s, \theta)\right\|_{\infty}=0.9840<1 .
$$

In order to illustrate the robustness of the system when the observer parameter $k$ is changed, in what follows, the stability condition (44) is analyzed. Consider time-delays uncertainties of $\theta_{1}=0.01$ and $\theta_{2}=0.04$. Under these conditions, Figure 10 shows condition (44) for different values of $k$. As it is seen, while the value of the parameter $k$ increase, the stability condition (44) tends to its limit. Notice that for $k=0.45$, condition (44) is not satisfied. From this analysis, it is possible to see that the robustness of the control strategy can be improved by properly choosing the observer parameter $k$. However, a compromise between performance and robustness is obtained.

As in the previous example, it is considered a step disturbance $D(s)=-0.05$ acting at $t=40 \mathrm{sec}$. Figure 11 shows the evolution of the output and control signals when considering a zero initial conditions (continuous line) and when 
the initial condition in the recycle path is set at 0.01 (dashed line). Figure 12 shows the error estimation of the recycle signal $e_{\omega_{2}}(t)$ when $D(s)=0$ and a small initial condition of magnitude 0.07 is present in the backward path process.

Lemma 4 guarantee that the controller asymptotically reject step disturbances d(t), i.e., to make the sensitivity $S(s)=0$ for $s=j w=0$.

It could be interesting to have a "small" sensitivity $S(j w)$ for a wide range of $w$ and not only for $w=0$, but from expression (26) it is clear that the sensitivity depends on controller parameters $k, K_{p}$ and $T_{i}$. Figure 13 illustrate $S(j w)$ for tree different values of $k$.

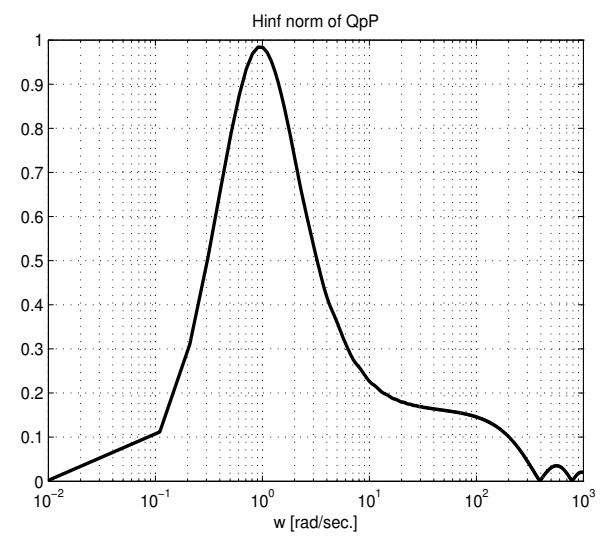

Figure 9: $\left\|N_{c}(s) D_{c}(s, \theta)\right\|_{\infty}$ for $\theta_{1}=0.016$ and $\theta_{2}=0.05$.

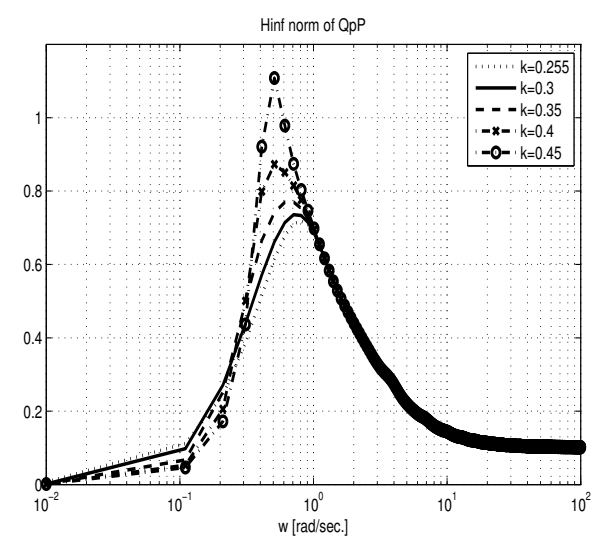

Figure 10: $\left\|N_{c}(s) D_{c}(s, \theta)\right\|_{\infty}$ for different values of $k$ with $\theta_{1}=0.01$ and $\theta_{2}=0.04$. 

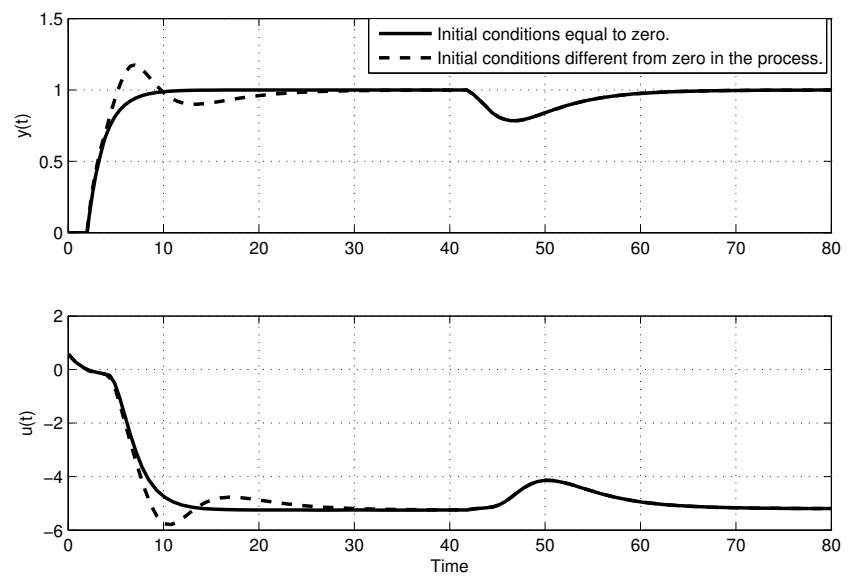

Figure 11: Output and control signals in Example 5.

\section{Conclusions}

Using recycle in unstable processes with significant time-delay leads to a challenging control problem. In this work this problem has been addressed for the particular case of one unstable pole with significant delay in the forward path. Explicit conditions for the construction of an stabilizing observer-based controller scheme for such class of systems are presented. The observer-prediction strategy is used to estimate some internal variables of the process required for: i) remove the dynamics of backward loop in the recycling process and ii) design a stabilizing control law for the free delay model in the forward path. The basis provided in this work could be useful for extending the class of systems for which the recycle can be used. Particularly, the case of one unstable pole with several stable poles plus significant time-delay in the forward path could be addressed. As it is well known, time-delay uncertainties can affect closed-loop stability of recycled systems, in order to prevent this possibility in the proposed controller, a robustness analysis has been developed. The proposed observer-based control scheme is evaluated by means of numerical simulations, showing an adequate response.

\section{References}

[1] B. Lehman. Stability of chemical reactions in a CSTR with delayed recycle stream. In American Control Conference, pages 3521-3522, Baltimore, Maryland, 1994.

[2] W. L. Luyben, B. D. Tyreus, and M. L. Luyben. Plantwide process control. McGraw-Hill, New York, 1998. 

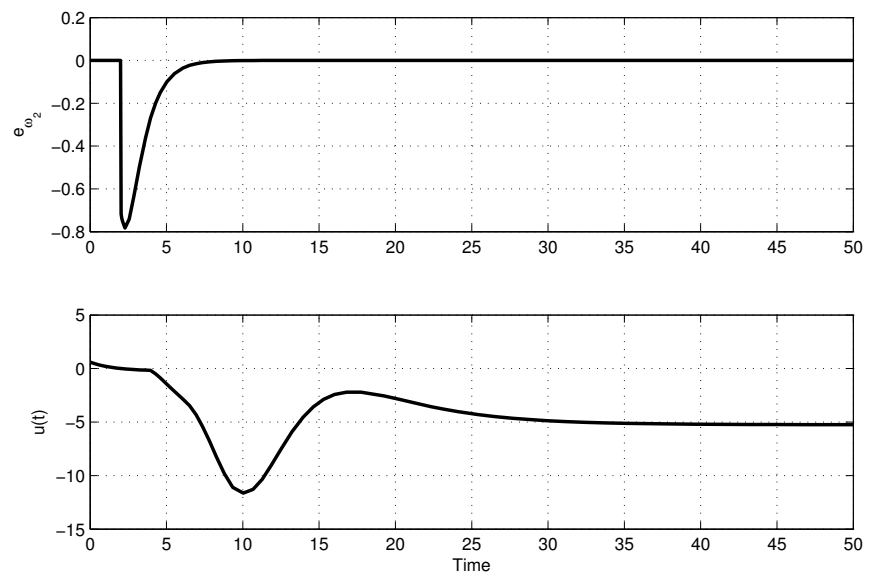

Figure 12: Estimation recycle error $e_{\omega}(t)$ and control signal $u(t)$, Example 5 .

[3] K. L. Wu and C. C. Yu. Reactor/separator processes with recycle-1. candidate control structure for operability. Comput. Chem. Eng., 20(11):12911316, 1996.

[4] C. S. Bildea and A. C. Dimian. Stability and multiplicity approach to the design of heat-integrated PFR. AIChe Journal, 44(12):2703-2712, 1998.

[5] C. S. Bildea, A. C. Dimian, and P. Iedema. Interaction between design and control of heat-integrated PFR. Computer Aided Chemical Engineering, 8:169-174, 2000.

[6] M. L. Luyben and M. L. Luyben. Essentials of process control. McGrawHill, 1996.

[7] W. L. Luyben. Temperature control of autorefrigerated reactors. J. Process Control, 9(4):301-312, 1999.

[8] S. Ibrir. Observer-based control of a class of time-delay nonlinear systems having triangular structure. Automatica, 47:388-394, 2011.

[9] X. G. Yan, S. K. Spurgeon, and C. Edwards. Sliding mode control for timevarying delayed systems based on a reduced-order observer. Automatica, 46:1354-1362, 2010.

[10] C. Hua, F. Li, and X. Guan. Observer-based adaptive control for uncertain time-delay systems. Information Sciences, 176:201-214, 2006.

[11] A. C. Dunn, L. S. Shieh, and S. M. Guo. Digital redesign of analog smith predictor for systems with input time delays. ISA Transactions, 43:33-47, 2004. 


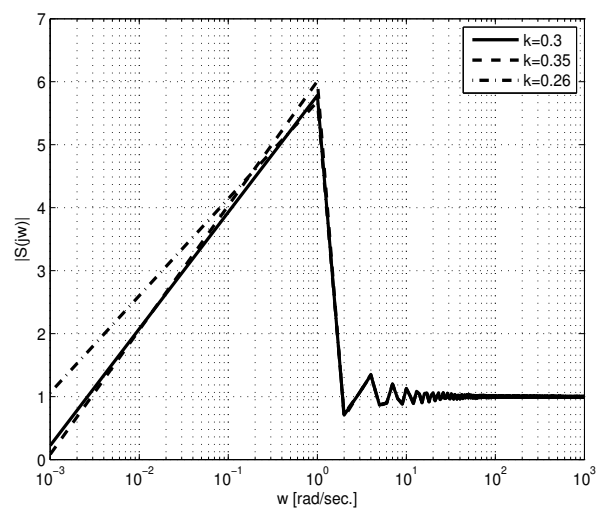

Figure 13: Sensitivity function in Example 5.

[12] A Papadourakis, M. F. Doherty, and J. M. Douglas. Approximate dynamic models for chemical process systems. Ind. Eng. Chem. Res., 28(5):546-552, 1989 .

[13] F. Gouaisbaut and D. Peaucelle. Stability of time delay systems with nonsmall delay. In Proccedings of the IEEE Conf. on Decision and control, pages 840-845, San Diego, CA, 2006.

[14] J. Zhang, C. R. Knospe, and P. Tsiotras. New results for the analysis of linear systems with time-invariant delays. Int. J. Robust Nonlin. Control, 13(12):1149-1175, 2003.

[15] K. E. Kwok, M. Chong-Ping, and G. A. Dumont. Seasonal model based control of processes with recycle dynamics. Ind. Eng. Chem. Res., 40(7):16331640, 2001.

[16] B. Del Muro-Cuéllar; M. Velasco-Villa; H. Puebla and J. Álvarez Ramírez. Model approximation for dead-time recycling systems. Ind. Eng. Chem. Res., 44(12):4336-4343, 2005.

[17] C. Scali and F. Ferrari. Performance of control systems based on recycle compensators in integrated plants. J. Process Control, 9(5):425-437, 1999.

[18] K. J. Astrom, C. C. Hang, and B. C. Lim. A new smith predictor for controlling a process with an integrator and long dead time. IEEE Trans. Aut. Control, 39(2):343-345, 1994.

[19] L. Maza-Casas, M. Velasco-Villa, and Ja. Alvarez-Gallegos. On the state prediction of linear systems with time-delays in the input and the state. In Proc. 38th. IEEE Conf. On Decision and Control, pages 239-244, USA, December 1999. 
[20] J. F. Márquez-Rubio, B. del Muro-Cuéllar, M. Velasco-Villa, and D. Cortes. Observer scheme for linear recycling systems with time delays. In American Control Conference, pages 4904-4909, San Francisco, CA, 2011.

[21] J. F. Márquez-Rubio, B. del Muro-Cuéllar, M. Velasco-Villa, and J. Álvarez Ramírez. Control based in an observer scheme for first-order system with delay (in spanish). Revista Mexicana de Ingeniería Química, 9(1):43-52, 2010 .

[22] J. K. Hale and S. M. Verduyn Lunen. Introduction to Functional Differential equations. Springer-Verlag, New York, 1993.

[23] G. J. Silva and S. P. Bhattacharyya. PID controllers for time-delay systems. Birkhuser, Boston, 2005.

[24] S. I. Niculescu. Delay Effects on Stability. A robust control approach. Spinger-Verlag, London, 2001.

[25] Y. Pedraza, O. González, and B. del Muro-Cuéllar. Stabilization of high order systems with delay using a predictor schema. In IEEE International Midwest Symposium on Circuits and Systems, Cancun, Mex., 2009.

[26] K. J. Astrom and T. Hagglund. PID Controllers, theory, design and tuning. International Society for Measurement and Control, New York, 1995.

[27] N. Levinson and R.M. Redheffer. Complex Variables. Holden-Day, Baltimore, USA, 1970.

[28] L. Dugard and E. I. Verriest (Eds). Stability and control of time-delay systems. Springer Verlag, New York, 1998.

[29] O. Sename and C. Briat. Observer-based $H_{\infty}$ control for time-delay systems: a new LMI solution. In IFAC TDS Conference, L'Aquila, Italy, 2006 .

[30] E. I. Verriest, O. Sename, and P. Pepe. Robust observer-controller for delay-differential systems. In Conference on Decision and Control, pages 981-986, La9 Vega, Nevada, USA, 2002.

[31] A. Weinmann. Uncertain models and robust control. Springer-Verlag, New York, 1991.

[32] Z. J. Palmor and Y. Halevi. Robustness properties of sampled-data systems with dead time compensators. Automatica, 26(3):637-640, 1990.

[33] B. W. Bequette. Process control: Modeling, design and simulation. Prentice Hall, 2003. 


\section{Appendix A. Proof of Lemma 1 and Corollary 1}

Proof of Lemma 1. The proof use the well known fact that a discrete time model derived from a continuous time system is equal to its continuous counterpart if the sampling period $T \rightarrow 0$ by considering a zero order hold device. It is carried out by discretizing the system and then showing that all the poles remain inside the unitary circle when the sampling period tends to zero iff $\tau<\frac{1}{a}$.

Discretizing model (6) using a zero order hold and a sampling period $T=\frac{\tau}{n}$ with $n \in \mathbb{N}$, it is obtained,

$$
G(z)=\frac{b}{a} \frac{\left(e^{a T}-1\right)}{z^{n}\left(z-e^{a T}\right)}
$$

Model (A.1) in closed-loop with the (discretized) output feedback (7) produces the characteristic equation,

$$
p(z)=z^{n}\left(z-e^{a T}\right)+k \frac{b}{a}\left(e^{a T}-1\right)=0
$$

Let us to analyze the root locus of (A.2). Open loop system has $n$ poles at the origin and one at $z=e^{a T}$. Then, there exist $n+1$ branches to infinity, $n-1$ of them starting at the origin and going directly to infinity. The two remaining branches starting at a breaking point $z_{1}$ located over the real axis between the origin and $z=e^{a T}$ (this situation is illustrated in Figure A.14 for the case $n=5)$. $z_{1}$ can be found by considering the equation,

$$
\frac{d k}{d z}=\frac{d}{d z}\left[-\frac{z^{n}\left(z-e^{a T}\right)}{\frac{b}{a}\left(1-e^{a T}\right)}\right]=0,
$$

producing the equation,

$$
(n+1) z^{n}-n z^{n-1} e^{a T}=0,
$$

which has $n-1$ roots at the origin and one at,

$$
z_{1}=\frac{n}{n+1} e^{a \frac{\tau}{n}} .
$$

If the breaking point $z_{1}$ over the real axis is located inside the unit circle, the closed-loop system could have a region of stability, otherwise the system is unstable for any $k$.

The stability properties of the continuous system (8) are alternately obtained by considering the limit as $n \rightarrow \infty$, or equivalently, when $T \rightarrow 0$ of equation (A.3), this is,

$$
\lim _{n \rightarrow \infty} z_{1}=\lim _{n \rightarrow \infty} \frac{n}{n+1} e^{a \frac{\tau}{n}}=1 .
$$

It is important to note that any point $s=\theta$, over the real axis on the complex plane $s$ is mapped to $z=e^{\theta T}$ on the $z$ plane and as a consequence this point 


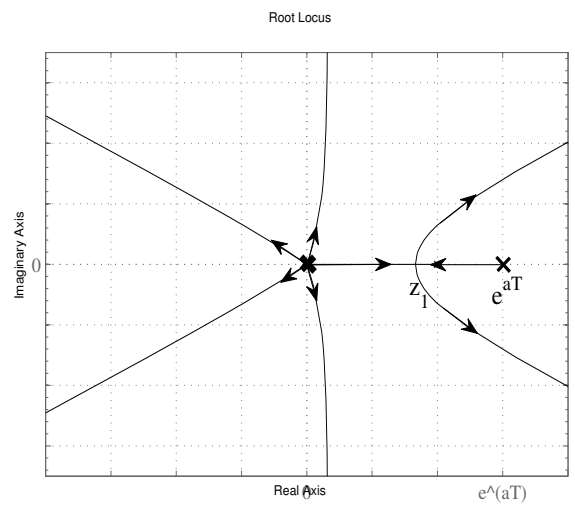

Figure A.14: Root locus of equation (A.2) for $n=5$.

converges to $z=1$ when $T$ tends to zero. Notice also that any real point $s=\theta$ on the left half side of the complex plane $(\theta<0)$ is mapped to a point $e^{\theta T}$ that tends to one over the stable region of the $z$ plane. On the contrary, if $\theta$ is on the right side of the complex plane over the real axis $(\theta>0)$, the point $e^{\theta T}$ tends to one over the unstable region. Then, from (A.2), it is not difficult to see that if $a \tau<1$ (i.e., $\tau<1 / a$ ) there exists a gain $k$ that stabilizes the closed-loop system (i.e., the limit tends to one from the left). In the case that $a \tau \geq 1$ it is not possible to get $k$ that stabilize the system.

Then, if the remaining $n-1$ roots are into the unit circle, the closed-loop is stable. Let us now prove that the remaining $n-1$ roots are into the unitary circle if and only if $a \tau<1$. Assume that $a \tau \leq 1$ and to take into account the continuous case, the characteristic equation (A.2) is modified as,

$$
\begin{aligned}
\lim _{n \rightarrow \infty} p(z) & =\lim _{n \rightarrow \infty}\left[z^{n}\left(z-e^{a T}\right)+k \frac{b}{a}\left(e^{a T}-1\right)\right] \\
& =\lim _{n \rightarrow \infty}\left[z^{n}\left(z-e^{a \frac{\tau}{n}}\right)+k \frac{b}{a}\left(e^{a \frac{\tau}{n}}-1\right)\right] \\
& =(z-1) \lim _{n \rightarrow \infty} z^{n}
\end{aligned}
$$

from where it is stated that while one pole is on the neighborhood of $z=1$, the remaining poles are in a neighborhood of the origin. Then, we can finally state that the system can be stabilized iff $a \tau<1$.

\section{Proof of Corollary 1.}

Assume that $\tau<\frac{1}{a}$ and take into account the discretized system given by (A.1). Analyzing the root locus associated to such discrete system, it is possible to see that the open loop system has $n$ poles at the origin and one at $z=e^{a T}$ without finite zeros. Then, there are $n-1$ branches going to infinity and a pair converging to a point on the real axis located between the origin and 
$z=1$ (stability region). Note that if $k=0$ the system is unstable. The gain $k$ that takes the systems to the border of the stability region $(z=1)$ is obtained by evaluating $k$ for $z=1$, this is,

$$
k=-\left.\frac{z^{n}\left(z-e^{a T}\right)}{\frac{b}{a}\left(1-e^{a T}\right)}\right|_{z=1}=\frac{a}{b}
$$

Then by Lemma 1 the proof is concluded. 